\title{
Travelling together: a unifying pathomechanism for ALS
}

Pietro Fratta ${ }^{1 \#}$, Nicol Birsa ${ }^{1,2}$, Andrew P. Tosolini ${ }^{1}$, Giampietro Schiavo ${ }^{1,2,3 \#}$

${ }^{1}$ Department of Neuromuscular Diseases, UCL Queen Square Institute of Neurology, University College London, London WC1N 3BG, UK.

2 UK Dementia Research Institute, University College London, London WC1E 6BT, UK.

${ }^{3}$ Discoveries Centre for Regenerative and Precision Medicine, University College London

Campus, London WC1N 3BG, UK.

"Correspondence to:

Pietro Fratta p.fratta@ucl.ac.uk

Giampietro Schiavo giampietro.schiavo@ucl.ac.uk

Keywords: amyotrophic lateral sclerosis; annexin A11; axonal transport; LAMP1-positive organelles; RNA granules

\begin{abstract}
Axonal transport is critical for neuronal homeostasis and relies on motor complexes bound to cargoes via specific adaptors. However, the mechanisms responsible for the spatiotemporal regulation of axonal transport are not completely understood. A recent study by Liao et al. contributes to filling this gap by reporting that RNA granules 'hitchhike' on LAMP1-positive organelles using annexin A11 as a tether.
\end{abstract}

\section{Main text}

Intracellular trafficking is paramount to ensure cell homeostasis and survival, and is particularly important in neurons due to their large size and complex morphology. In axons, this process is driven by microtubule-dependent motor complexes, which are connected to their cargo by specific adaptors. Local availability of motor adaptors (e.g., via local synthesis) or their activation by posttranslational modification is thought to dictate motor complex recruitment and the overall efficiency of axonal transport, both in terms of frequency and speed of the transported cargoes [1]. However, it is unclear how cargo diversity is recognised by a limited number of adaptor proteins. Furthermore, the mechanisms enabling membraneless organelles, such as RNA granules, to undergo long-range transport is completely unknown.

These gaps are now starting to be addressed by expanding and further refining the concept of organelle hitchhiking originally proposed by work in fungi [2]. This seminal study, by Gero Steinberg and colleagues, demonstrated that a variety of organelles, including peroxisomes, lipid droplets, and endoplasmic reticulum, share a ride on motile early endosomes.

Most recently, Liao et al. found that RNA granules hitchhike on different organelles including LAMP1-, Sec61- and Rab7-positive vesicles in cell lines, and in axons of rat cortical neurons [3]. These organelles use annexin A11 (ANXA11) as a molecular tether, which provides a reversible interaction between the primary transported cargo (a LAMP1-positive late endosome/lysosome) and an RNA granule. Indeed, upon heat shock, ANXA11 bridges 
LAMP-1 positive organelles with G3BP1 granules through membrane binding and phase separation properties, respectively.

This cunning (and largely unexpected) mechanism ensures an overall economy of scale by linking a pool of molecular motors to two different cargoes, thus enabling their coordinated transport. It also allows the combinatorial pairing of diverse organelles, which could be coupled in a spatiotemporal manner to respond to precise functional demands, such as, for example, local translation and/or specific energetic needs [4]. Given the low percentage of LAMP1-positive organelles found associated with G3BP1-containing RNA granules [3], and the high selectivity of ANXA11 in tethering these two cargoes, it is unlikely that this is the only molecular tethering mechanism used in axons. Indeed, determining whether G3BP1-negative RNA granules also exploit an ANXA11-dependent tethering mechanism, and whether all axonal RNA delivery relies on a combinatorial array of diverse tethers and transport organelles, will be one of the logical follow-ups to these experiments. Furthermore, as neuronal LAMP1-positive organelles are very diverse, and comprise not only mature, degradative lysosomes, but also various intermediates of the endocytic and autophagic pathways [5], determining the specificity of the pairing between RNA granules and subtypes of LAMP1-positive carriers will also be of paramount importance.

However, perhaps the most important contribution of the work by Liao et al. is providing the field with a novel pathomechanism of ALS that unifies work from two streams of research, which have been disconnected to date. On the one hand, mutations in components of the axon transport machinery and dysfunctions of axonal transport have been described in ALS and in other neurodegenerative diseases [1]. On the other hand, and independently, genetic evidence has highlighted the role of ALS mutations in impairing the dynamics of membraneless organelles. For example, mutations in the RNA binding proteins TDP-43, FUS and hnRNP A1 directly alter the liquid-liquid phase separation (LLPS) that drives the formation of these organelles, and mutations in other ALS genes, such as VCP, impact on their disassembly.

The work of Liao et al. demonstrated that ANXA11 mutations, recently identified in familial cases of ALS [6], impair RNA granule transport by altering the link of these membraneless organelles to LAMP1-positive carriers. Therefore, the functional consequences of ALS mutations for components of the machinery enabling transport and unloading of RNA at specific neuronal locations, can now be mechanistically linked. Local disassembly of organelles in axons has been shown to be important in regulating local protein translation, which is essential for axonal maintenance and response to injury. Alterations in local protein synthesis within specific segments of the axon and/or at the neuromuscular junction, could contribute to axonal dyshomeostasis, ultimately leading to denervation and motor neuron death. Furthermore, the functional association between LAMP1-positive organelles and RNA granules implies that axonal transport deficits, caused by different axonal insults (e.g. the proximity to an amyloid plaque), would also cause alterations in RNA transport causing neurodegeneration.

An outstanding question is how protein translation is regulated in axons. Posttranslational modifications, such as phosphorylation of RNA binding proteins in RNA granules, which could be induced by local stimuli, have been shown to regulate granule disassembly and enhance translation [7]. However, whether granules need to uncouple from the tethered organelles, or whether translation occurs while they are stably linked to organelles, as previously shown for Rab7 endosomes [4], is currently unknown.

Although RNA transport and local translation occur in all cell types, neurons may rely more strictly on this finely-tuned mechanism due to their extreme size and morphology, and as a consequence, may be more vulnerable than other cells to perturbations in these processes. An enhanced understanding of cell specific RNA and protein composition of membraneless transport organelles may contribute to address this open question, as well as to clarify why distinct neuronal subtypes are differentially susceptible to these disruptions. 
The recent demonstration that Charcot Marie Tooth (CMT) disease-associated mutations in Rab7, a small GTPase often associated to LAMP1-positive organelles and essential for the axonal transport of signalling endosomes [1], impact on axonal translation [4], further highlights the importance of this process in the maintenance and function of peripheral axons, and widens our understanding of the pathogenesis of this hereditary neuropathy. Remarkably, the CMT phenotype is much slower than ALS and involves the sensory system in addition to motor axons, highlighting the additional still poorly defined mechanisms that are at play in ALS, including TDP-43 proteinopathy. In this regard, whilst rare TDP-43 mutations can alter the transport of RNA [8], the majority of ALS cases share accumulation of TDP-43 in cytoplasmic aggregates and its depletion from the nucleus. Recent evidence shows that nuclear loss of function of TDP-43 induces toxicity through altered splicing and reduction of stathmin-2 [9, 10], which in turn has important roles in axonal function and regeneration, and may therefore exacerbate the aforementioned axonal dysfunction.

Altogether, these exciting discoveries open the possibility to further test this integrated pathomechanism for ALS, which would in turn enable the identification of novel and more effective drug targets for this incurable disease.

\section{Acknowledgements}

This work was supported by a Medical Research Council Senior Fellowship award [PF and NB], the UCLH NIHR Biomedical Research Centre [PF], the European Union's Horizon 2020 Research and Innovation programme under grant agreement 739572 [GS], a Wellcome Trust Senior Investigator Award (107116/Z/15/Z) [GS and APT] and a UK Dementia Research Institute Foundation award [GS].

\section{References}

1. Sleigh, J.N. et al. (2019) Axonal transport and neurological disease. Nat Rev Neurol.

2. Guimaraes, S.C. et al. (2015) Peroxisomes, lipid droplets, and endoplasmic reticulum "hitchhike" on motile early endosomes. J Cell Biol 211, 945-54.

3. Liao, Y.C. et al. (2019) RNA granules hitchhike on lysosomes for long-distance transport, using annexin A11 as a molecular tether. Cell 179, 147-164 e20.

4. Cioni, J.M. et al. (2019) Late endosomes act as mRNA translation platforms and sustain mitochondria in axons. Cell 176, 56-72 e15.

5. Cheng, X.T. et al. (2018) Characterization of LAMP1-labeled nondegradative lysosomal and endocytic compartments in neurons. J Cell Biol 217, 3127-3139.

6. Smith, B.N. et al. (2017) Mutations in the vesicular trafficking protein annexin A11 are associated with amyotrophic lateral sclerosis. Sci Transl Med 9.

7. Sahoo, P.K. et al. (2018) Axonal G3BP1 stress granule protein limits axonal mRNA translation and nerve regeneration. Nat Commun 9, 3358.

8. Alami, N.H. et al. (2014) Axonal transport of TDP-43 mRNA granules is impaired by ALScausing mutations. Neuron $81,536-543$.

9. Melamed, Z. et al. (2019) Premature polyadenylation-mediated loss of stathmin-2 is a hallmark of TDP-43-dependent neurodegeneration. Nat Neurosci 22, 180-190.

10. Klim, J.R. et al. (2019) ALS-implicated protein TDP-43 sustains levels of STMN2, a mediator of motor neuron growth and repair. Nat Neurosci 22, 167-179. 